\title{
Análisis de los objetivos del mercado de trabajo y de capital humano y tecnológico de la Estrategia de Lisboa por Comunidades Autónomas. Un indicador de cumplimiento
}

\author{
M. ${ }^{a}$ Cruz Merino Llorente \\ Universidad de Valladolid. Facultad de Ciencias del Trabajo \\ Dpto. Economía Aplicada. Área de Economía Aplicada \\ Avda. de Madrid, 44. 34004 Palencia (España) \\ mcmerino@ea.uva.es
}

\section{M. ${ }^{a}$ Noelia Somarriba Arechavala \\ Universidad de Valladolid. Facultad de Ciencias del Trabajo \\ Dpto. Economía Aplicada. Área de Estadística y Econometría Avda. de Madrid, 44. 34004 Palencia (España) \\ nsomarri@eco.uva.es}

\begin{abstract}
RESUMEN
Basándonos en los objetivos planteados en la Estrategia de Lisboa, en el Consejo Europeo de Primavera de marzo de 2005 y en el Programa Nacional de Reformas, en relación con la productividad y el empleo, el propósito de esta comunicación es diseñar un instrumento a nivel regional que nos permita evaluar el progreso en el cum-

* Las autoras agradecen los comentarios y sugerencias del evaluador anónimo que han contribuido a mejorar este trabajo.
\end{abstract}


plimiento de los objetivos de la Estrategia de Lisboa en materia de empleo, capital humano e I+D en las distintas Comunidades Autónomas. Para ello, se parte de un conjunto de indicadores estructurales, con el objetivo de estimar un indicador regional de cumplimiento global por medio de la medida de distancia $\mathrm{P}_{2}$.

Palabras clave: empleo, capital humano, inversión y desarrollo, Estrategia de Lisboa.

JEL: E61, R23.

\section{INTRODUCCIÓN}

El objetivo de este artículo es el análisis de la situación regional de los indicadores estructurales en materia de empleo, capital humano e investigación y desarrollo (I+D), desarrollados por la Comisión Europea, con los datos disponibles más recientes, con el fin de poder evaluar el avance de las Comunidades Autónomas en términos de cumplimiento de los principales objetivos del Programa Nacional de Reformas (y en su caso, de la Estrategia de Lisboa) y compararla con la media nacional.

En el Consejo Europeo de Lisboa de marzo de 2000, la Unión Europea acuerda como objetivo estratégico para el año 2010 «convertirse en la economía basada en el conocimiento más competitiva y dinámica del mundo, capaz de crecer económicamente de manera sostenible con más y mejores empleos y con mayor cohesión». Detrás de este ambicioso objetivo está la consecución de un mayor nivel de bienestar europeo compatible con la conservación y ampliación de la cohesión social; incidiendo, por tanto, en las dimensiones económica y social del modelo de crecimiento esperado.

Tras la evaluación intermedia de la Estrategia de Lisboa a mitad de camino, con resultados poco satisfactorios, el Consejo Europeo de primavera de 2005 contempla la necesidad de reformar y relanzar la Estrategia, reduciendo y simplificando la lista de objetivos y prioridades, concentrando mayores esfuerzos y recursos en aquellas áreas que tengan un mayor impacto sobre el crecimiento y el empleo (bautizada como Estrategia Renovada de Lisboa de Crecimiento y Empleo). La presentación por parte de los Estados miembros del Programa Nacional de Reformas (en adelante, PNR) supone un mayor compromiso político de los gobiernos en esa dirección; en el caso español, su PNR establece como objetivos principales alcanzar la plena convergencia en renta per cápita con la Unión Europea en 2010 y alcanzar una tasa de empleo del $66 \%$, lo que requiere, además de incrementar la participación laboral, un aumento de la productividad a un ritmo similar al europeo.

El interés de este artículo radica en el estudio de la situación de las diferentes 
Comunidades Autónomas en áreas trascendentales para su crecimiento como son el empleo, el capital humano y la I+D, así como en la propuesta de algunas medidas necesarias para el logro de los objetivos de la Estrategia de Lisboa y de esta forma corregir ciertas debilidades regionales. Además la elaboración de indicadores sintéticos de cumplimiento permite la realización de comparaciones a nivel espacial, reflejando el camino que le queda por recorrer a cada Comunidad Autónoma en relación a los objetivos de Lisboa y cuál es su realidad en relación con el conjunto nacional.

Tras esta introducción, en el siguiente apartado se selecciona un conjunto de indicadores estructurales que permitirán analizar y valorar el avance conseguido en los objetivos a nivel regional. Los indicadores elegidos muestran el estado de diversos aspectos del mercado de trabajo y de la inversión en capital tecnológico y humano. Después de una descripción de la metodología de la medida de distancia $\mathrm{P}_{2}$, se realiza un breve análisis de la situación de las diferentes Comunidades Autónomas respecto a estos indicadores parciales. A continuación, se aplica la metodología de la $\mathrm{P}_{2}$, con la finalidad de obtener un indicador de cumplimiento global de los objetivos de Lisboa en materia laboral y de capital humano (IG) y dos indicadores individualizados para el capital humano (ICH) y para el área del mercado de trabajo (IL), finalizando con algunas reflexiones sobre los resultados y un apartado de conclusiones.

\section{SELECCIÓN DE INDICAdORES}

Las variables seleccionadas, con información disponible a nivel regional, son indicadores relativos al mercado de trabajo, al capital humano y a la investigación y al desarrollo; áreas necesarias para incrementar el potencial de crecimiento y así mejorar el PIB per cápita. Los principales indicadores se refieren a aspectos de la economía española que presentan determinadas debilidades que se desean corregir, con el propósito de fomentar el crecimiento y la competitividad.

Para alcanzar los objetivos del PNR español es necesario insistir en la creación de

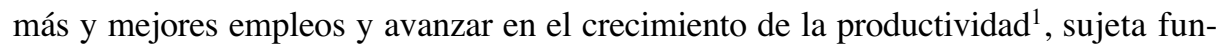
damentalmente a la inversión en capital físico, humano e intelectual, que debe mejorarse tanto en cantidad como en calidad. Se pretende con ello, por un lado, aumentar

${ }^{1}$ El crecimiento experimentado por la economía española, muy superior al de nuestros socios comunitarios, no ha permitido la total convergencia con la Unión Europea, en buena parte por el aumento de población en España y, por otra, porque el aumento notable de 
la participación en el mercado laboral y las oportunidades de empleo para determinados colectivos prioritarios y así evitar su exclusión social²:

- las mujeres, a través de medidas que permitan una mejor conciliación entre la vida laboral y personal, para lo que es fundamental la provisión de servicios de asistencia y cuidado de los menores de tres años y de las personas dependientes, y que se elimine la discriminación laboral y asegure la igualdad de género, tanto en términos de empleo, paro y remuneración;

- los trabajadores de más edad, para que continúen activos y disuadirles del abandono anticipado del mercado laboral y así prolongar su vida laboral, a través de acciones de formación, incentivos financieros y el fomento de unas condiciones de trabajo adecuadas para el envejecimiento activo ${ }^{3}$;

- y los jóvenes, con un notable retraso en la edad media de inserción al mercado laboral, debido a la prolongación de su período formativo, pocas veces simultaneado con el trabajo o la búsqueda de empleo, por lo que les resulta relativamente difícil encontrar empleo, de ahí el elevado paro juvenil. Para reducir su tasa de paro $^{4}$, se debería fomentar el trabajo a tiempo parcial para que puedan

empleo ha ido acompañado de un lento avance de la productividad del trabajo. En otras palabras, España está creciendo y generando empleo, pero aún no está mejorando como sería necesario en términos de productividad.

${ }^{2}$ En este sentido, en la evaluación de la ejecución de la Estrategia de Lisboa renovada, se hace referencia a la aplicación del concepto de flexiguridad, que pretende proteger al trabajador (en lugar de los puestos de trabajo), ayudándole a prepararse, a adaptarse ante los cambios rápidos, para así tener seguridad en el empleo.

${ }^{3}$ El aumento de la participación en el mercado laboral de los trabajadores de más edad es fundamental para apoyar el crecimiento económico y los sistemas de protección social. Se persigue un aumento de su tasa de empleo ya que, frente a la reducción prevista del número de trabajadores en edad laboral, constituyen un colectivo importante en la oferta de trabajo. En este sentido, se ha aprobado el 22 de noviembre de 2007 en el Congreso de los Diputados la propuesta de reforma de la Seguridad Social, en la que, entre otros puntos, se incluyen incentivos para prolongar la edad de jubilación, con la finalidad de reforzar la viabilidad futura del sistema de pensiones.

${ }^{4}$ Esta tasa se ha reducido como consecuencia de que los jóvenes constituyen el grupo que más sufre la temporalidad, con contratos temporales de corta duración y, por tanto, con una gran rotación laboral. 
compatibilizar ambas actividades o también garantizar a todo joven un período de prácticas como primer contacto con el mundo laboral.

Otro aspecto relevante de la Estrategia de Lisboa recae en las políticas de educación y formación. Se ha de invertir más en capital humano, mediante la mejora de las cualificaciones y las competencias de los trabajadores, modificando los sistemas de educación y de formación, dado que constituyen un elemento básico en la obtención de un puesto de trabajo, en la adaptación ante cambios rápidos y en el aprovechamiento de las nuevas tecnologías. Se incide mucho en la formación continua a lo largo de toda la vida, a través de técnicas de aprendizaje permanente accesibles a todas las personas en las escuelas, universidades y empresas, que les permitan obtener las competencias necesarias ante cualquier perturbación.

En cuanto a la innovación y a la I+D, la innovación es un determinante clave del crecimiento económico a más largo plazo; se deben mejorar los servicios de apoyo a la innovación, en concreto, para la difusión y la transferencia de tecnología, la constitución de grupos de innovación, mejoras en el acceso a la financiación nacional e internacional. Es prioritario aumentar y mejorar la inversión en I+D, fundamentalmente por parte del sector empresarial, para aumentar la productividad a medio plazo y promover y avanzar en la difusión y el uso de las tecnologías de la información y la comunicación (TIC) ${ }^{5}$.

Los indicadores que se incluyen en nuestro indicador sintético, para poder diseñar el indicador regional de evaluación del cumplimiento de los objetivos de la Estrategia de Lisboa, son los que aparecen en el cuadro 1, donde también se recogen los objetivos que establece el PNR (y en su ausencia los establecidos en la Estrategia de Lisboa), el ámbito temporal, el dato correspondiente a España y el número de Comunidades Autónomas que cumplen ya el objetivo establecido para el 2010.

${ }^{5}$ La Comisión Europea destaca que uno de los puntos fuertes del PNR español es la puesta en marcha del programa INGENIO 2010 para fomentar la I+D+i (cuarto eje de actuación). 
Cuadro 1. Indicadores de mercado de trabajo, educación e I+D

\begin{tabular}{|c|c|c|c|c|c|}
\hline Indicador & Objetivo PNR & Objetivo UE & $\begin{array}{l}\text { Fecha del } \\
\text { dato más } \\
\text { reciente* }\end{array}$ & $\begin{array}{c}\text { Dato medio } \\
\text { de } \\
\text { España }\end{array}$ & $\begin{array}{l}\mathrm{N}^{0} \text { de CCAA } \\
\text { que cumplen } \\
\text { el objetivo }\end{array}$ \\
\hline Tasa de crecimiento del PIB & $3 \%$ & $3 \%$ & 2006 & $3,9 \%$ & 17 \\
\hline \multicolumn{6}{|l|}{ MERCADO DE TRABAJO } \\
\hline Tasa de empleo ${ }^{(1)}$ & $66 \%$ & $70 \%$ & 2006 & $65,75 \%$ & 8 \\
\hline Tasa de empleo femenino & $57 \%$ & $60 \%$ & 2006 & $53,97 \%$ & 6 \\
\hline $\begin{array}{l}\text { Tasa de empleo de los } \\
\text { trabajadores de } 55 \text {-64 años }\end{array}$ & $50 \%$ & $50 \%$ & 2006 & $47,25 \%$ & 5 \\
\hline Tasa de paro juvenil & $18,6 \%$ & & 2006 & $17,92 \%$ & 12 \\
\hline Siniestralidad laboral ${ }^{(2)}$ & $\nabla 15 \%$ & & 2005 & 6.011 & 2 \\
\hline \multicolumn{6}{|c|}{ EDUCACIÓN, I+D, NUEVAS TECNOLOGÍAS } \\
\hline Gasto en I+D (\% PIB) & $2 \%$ & $3 \%$ & 2005 & 1,1 & 0 \\
\hline $\begin{array}{l}\text { Participación privada en el } \\
\text { gasto en I+D }\end{array}$ & $55 \%$ & 2/3 & 2005 & 53,66 & 6 \\
\hline $\begin{array}{l}\text { Conexión a Internet en las } \\
\text { escuelas }^{(3)}\end{array}$ & $100 \%$ & $100 \%$ & Curso 2004-05 & $98,5 \%$ & 2 \\
\hline $\begin{array}{l}\text { Penetración de Internet } \\
\text { en los hogares }\end{array}$ & $30 \%$ & $30 \%$ & 2006 (1er semestre) & $35,9 \%$ & 16 \\
\hline Nivel educativo ${ }^{(4)}$ & $80 \%$ & $85 \%$ & 2005 & $62,4 \%$ & 1 \\
\hline Escolarización a los 2 años & $>30 \%$ & & Curso 2003-04 & & 4 \\
\hline $\begin{array}{l}\text { Tasa bruta de graduación } \\
\text { en ESO }\end{array}$ & $>80 \%$ & & Curso 2002-03 & $70,3 \%$ & 2 \\
\hline Abandono escolar prematuro ${ }^{(5)}$ & $15 \%$ & $10 \%$ & 2005 & $30,8 \%$ & 1 \\
\hline $\begin{array}{l}\text { Graduados superiores en } \\
\text { ciencias, matemáticas y } \\
\text { tecnología }{ }^{(6)}\end{array}$ & $13,5 \%$ & $15 \%$ & Curso 2002-03 & $12,2 \%$ & 8 \\
\hline Formación permanente ${ }^{(7)}$ & $12,5 \%$ & $12,5 \%$ & $2005\left(2^{\circ}\right.$ trimestre $)$ & $12,1 \%$ & 6 \\
\hline
\end{tabular}

* No podemos diseñar un indicador más homogéneo desde el punto de vista temporal, ya que los datos del Ministerio de Educación en algunos de los indicadores corresponden al curso académico 2002-03.

(1) Porcentaje de ocupados de entre 15 y 64 años sobre la población del mismo tramo de edad, que en el caso español el tramo se calcula entre 16 y 64 años.

(2) El índice de incidencia se define como el ratio accidentes de trabajo con baja en jornada laboral por 100.000 trabajadores afiliados a la Seguridad Social.

(3) Porcentaje de centros con conexión a Internet.

(4) Porcentaje de población de 20 a 24 años que ha completado, al menos, el segundo ciclo de la educación secundaria.

${ }^{(5)}$ Proporción de personas de 18 a 24 años que han abandonado prematuramente el sistema educativo.

(6) Número de graduados superiores en ciencias, matemáticas y tecnología por 1.000 habitantes de la población de 20 a 29 años.

(7) Población de 25 a 64 años (ocupados y no ocupados), que en las cuatro últimas semanas ha cursado algún tipo de estudios, bien en el marco de la empresa/centro de trabajo o fuera de él.

Fuente: Elaboración propia. 
La información necesaria para el cálculo de estos indicadores procede de distintas fuentes. La Contabilidad Regional (INE) nos permite disponer de la tasa de crecimiento del PIB, mientras que las variables del mercado laboral y de la formación permanente proceden de la Encuesta de Población Activa (EPA) ${ }^{6}$. Los datos de siniestralidad se obtienen del Ministerio de Trabajo y Asuntos Sociales. El resto de los indicadores de capital humano y la conexión de Internet en las escuelas proceden del Sistema Estatal de Indicadores de la Educación 2006 del Ministerio de Educación.

El gasto en $\mathrm{I}+\mathrm{D}$ es proporcionado por la Estadística sobre Actividades de I+D (INE, 2005), mientras que los datos relativos a la participación privada en la financiación de ese gasto se derivan de la Encuesta sobre Innovación Tecnológica en las Empresas (INE, 2005). Por último, la información de la penetración de Internet en los hogares procede de la Encuesta sobre Equipamiento y Uso de Tecnologías de la Información y Comunicación en los Hogares (INE, 2006).

\section{Metodología: la medida de distancia $\mathrm{DP}_{2}$}

Los indicadores sintéticos tienen como principal ventaja que condensan en una sola variable una gran cantidad de información, facilitando y permitiendo la realización de comparaciones espaciales y temporales. Tal y como establece Pena (1977): «se entiende por indicador sintético o global aquella función matemática de los indicadores parciales que reúne un conjunto de condiciones que se juzgan necesarias para concretar una medición expresiva del objetivo buscado». Una de las mayores problemáticas que surgen en torno a la construcción de indicadores sintéticos guarda relación con el diseño e implementación de esa función matemática, a la que se hace referencia en la definición y que agrega a los indicadores parciales de forma que se obtenga un indicador sintético global.

Las medidas de distancia, en general, constituyen una alternativa muy habitual en el diseño de indicadores sintéticos. Como representación de este grupo de medidas se ha seleccionado el índice $\mathrm{P}_{2}$ ya que verifica una serie de propiedades muy interesantes.

${ }^{6}$ La disponibilidad de los indicadores de empleo desagregados por sexo y tramos de edad permite valorar la situación concreta de estos colectivos que son prioritarios a la hora de intervenir y discernir las posibles actuaciones necesarias para el logro de los objetivos. 
La distancia $\mathrm{P}_{2}$, definida por el Profesor Pena Trapero (1977), es un indicador sintético que agrega la información contenida en un conjunto de indicadores sociales y que está diseñada para realizar comparaciones interespaciales e intertemporales.

Para un estudio exhaustivo del indicador $\mathrm{DP}_{2}$ se puede consultar Pena Trapero (1977) y posteriormente Zarzosa Espina (1996).

Se define la $\mathrm{DP}_{2}$ de la siguiente forma:

$$
D P_{2 j}=\sum_{i} \frac{d_{i}}{\sigma_{i}}\left(1-R_{i, i-1, i-2, \ldots, 1}^{2}\right) \operatorname{con} R_{i}^{2}=0
$$

donde:

- $m$ es el número de Comunidades Autónomas, $j=1, \ldots, m$

- $n$ es el número de indicadores, $i=1, \ldots, n$

- $x_{i j}$ es el valor del indicador $i$ en la Comunidad $j$

- $\sigma_{i}$ es la desviación típica del indicador $i$

- $R_{i, i-1, i-2, \ldots, 1}^{2}$ es el Coeficiente de Determinación en la Regresión de $X_{i}$ sobre $X_{i-1}$, $X_{i-2}, \ldots, X_{1}$

- $d_{i}$ es la distancia entre dos unidades, si la comparación se realiza entre ellas $d_{i}=d_{i}(\mathrm{r}, \mathrm{k})=\left|x_{r i}-x_{k i}\right|$; en el caso de que se este midiendo la situación de la unidad $\mathrm{r}$ con respecto a la base de referencia $X_{*}, d_{i}=d_{i}(\mathrm{r}, *)=\left|x_{r i}-x_{* i}\right|$.

- El cociente $d_{i} / \sigma_{i}$ mide la distancia entre las unidades a comparar ( $r$ y $k$ ) o la de una de ellas a la base de referencia $X_{*}$. Al dividirlo por $\sigma_{i}$ hace que las distancias se midan tipificadas, en el sentido de que retienen como unidad de medida las desviaciones típicas. De esta forma, las distancias de cada componente vienen expresadas en unidades abstractas y tiene sentido la aditividad. Además $\sigma_{i}$ puede interpretarse como una ponderación, de modo que las distancias entre dos países para cada componente, vienen ponderadas por la inversa de $\sigma_{i}$, de manera que la contribución de cada $\mathrm{d}_{\mathrm{i}}$ al indicador global es inversamente proporcional a la desviación típica del componente respectivo.

- El factor $\left(1-R_{i, i-1, i-2, \ldots, 1}^{2}\right)$ se denomina factor corrector y evita las redundancias al eliminar de los indicadores parciales la información ya contenida en los indicadores precedentes, es decir evita la duplicidad de información. El coeficiente de determinación $R_{i, i-1, i-2, \ldots, 1}^{2}$ mide la parte de la varianza de cada variable explicada por la regresión lineal realizada por las variables previas.

- En este caso de estudio, el indicador $\mathrm{DP}_{2}$ nos devolverá las distancias de cada 
Comunidad Autónoma respecto a una base de referencia, que estará constituida por los principales objetivos del PNR y en su defecto, de la Estrategia de Lisboa.

Este indicador presenta como gran ventaja que resuelve un gran número de problemas: la agregación de variables expresadas en distintas medidas, la ponderación arbitraria y la duplicidad de información. Estos problemas se solucionan a partir de este método de distancia, ya que el orden de entrada de los indicadores parciales, que condicionará el peso relativo de cada variable, se determina a través de un algoritmo que alcanza la convergencia cuando el indicador cumple una serie de propiedades deseables. Además, por medio de un mecanismo corrector, tan sólo se retiene de cada variable la información nueva que ésta incorpora, aprovechando la información útil y evitando la información duplicada.

Con las variables comentadas anteriormente se procede al cálculo del indicador sintético, para ello se ha empleado un algoritmo implementado en Matlab.

A raíz del cumplimiento de una serie de propiedades del indicador sintético, ciertas variables cuyo aumento implican un alejamiento de los objetivos se multiplicaron por -1 de forma que un aumento del valor de cualquier indicador suponga una menor distancia a los objetivos.

A la hora de obtener nuestro indicador sintético de distancia, como paso previo procederemos a normalizar las variables, el proceso de normalización consiste en:

$$
x_{n o r m i}=\frac{x_{j}-\min }{o b j-\min }
$$

donde

- $x_{i}$ es el valor del indicador de la comunidad $i$

- $\min$ es el peor resultado del indicador

- obj es el objetivo marcado

Aquellas Comunidades que han alcanzado el objetivo se les asignará el valor 1, de forma que nuestros indicadores quedarán normalizados entre 0 y 1 , donde 1 significa que la unidad de análisis ha verificado el objetivo y 0 que es la más alejada. En este caso la base de referencia estaría constituida por el vector unitario. 
En las tablas de resultados se incorpora el resultado del indicador sintético para España con el objetivo de de estudiar la distancia de las diferentes Comunidades Autónomas respecto al conjunto nacional ${ }^{8}$.

Teniendo en cuenta la tabla con los indicadores y los objetivos, dado que todas las Comunidades verifican el objetivo de la tasa de crecimiento del $\mathrm{PIB}^{9}$, el vector que recoge la variable normalizada sería el unitario con una desviación típica de 0 , por ello se omite esta variable de nuestra batería de indicadores ${ }^{10}$ para el cálculo de la $\mathrm{DP}_{2}$.

\section{Situación de las Comunidades Autónomas respecto a los indicadores parciales}

En este apartado se procede a realizar algunos comentarios sobre la situación de las Comunidades Autónomas respecto a los objetivos de la Estrategia de Lisboa en términos de los indicadores parciales, ello podrá arrojar algo de luz a la hora de interpretar los resultados de nuestros indicadores sintéticos que se presentan en la sección posterior.

\section{Indicadores del mercado de trabajo}

En cuanto a la tasa de empleo global, la media nacional en 2006 (65,75\%) casi alcanza el objetivo perseguido en el PNR para 2010 (del 66\%). Son muchas las Comunidades Autónomas que superan este objetivo (Aragón, Baleares, Cataluña, Comunidad Valenciana, Madrid, Navarra, País Vasco y la Rioja). Este grupo, excep-

${ }^{8}$ El dato para España se obtiene agregando el resultado de los indicadores sintéticos de las diferentes Comunidades Autónomas empleando como ponderaciones la población de cada Comunidad Autónoma entre la población para el total de España:

$w_{i} / w$ donde $w_{i}$ es la población de la Comunidad Autónoma $i$ y $w$ es la población para el total de España. Se han empleado los datos de población suministrados por el INE para el año 2006.

${ }^{9}$ En cuanto al objetivo estratégico del PIB per cápita establecido en el PNR, de alcanzar un nivel de bienestar social similar a la media comunitaria en 2010, cabe señalar que en 2006 hay cuatro regiones españolas que ya alcanzan el promedio del PIB de la UE-25, Madrid, País Vasco, Navarra y Cataluña. Sin embargo, la media nacional es inferior al nivel comunitario, estando por encima de la media además Baleares, Aragón y La Rioja.

${ }^{10}$ Las desviaciones típicas de los indicadores tienen que ser diferentes de cero en el cálculo de la $\mathrm{DP}_{2}$. 
to la Comunidad Valenciana, Aragón y el País Vasco, ya sobrepasa el objetivo ambicioso de la Estrategia de Lisboa de alcanzar el 70\% de tasa de empleo para 2010. En el lado opuesto se encuentran el resto de las regiones, destacando Extremadura, Andalucía y Asturias, como las más alejadas del objetivo del PNR, entre casi 10 y 7 puntos de diferencia.

El mercado de trabajo español presenta ciertas rigideces que impiden su funcionamiento eficiente. Aunque en los últimos años se ha creado mucho empleo, uno de sus problemas es la situación laboral de ciertos colectivos, como son las mujeres y los jóvenes.

En cuanto al empleo femenino, la posición de las Comunidades Autónomas respecto a la tasa de empleo femenino es similar que en el caso de la tasa de empleo global, aunque los datos son relativamente peores y queda más por avanzar. Es necesario seguir impulsando políticas y acciones de promoción laboral de la mujer para lograr el objetivo fijado; se debe continuar fomentando su participación laboral y la disminución del paro femenino, pues actualmente su tasa de paro duplica a la masculina. En la posición más desfavorecida se encuentra Extremadura, a más de 14 puntos del objetivo marcado. Del grupo mejor situado se desbanca la Comunidad Valenciana, con casi 2,5 puntos de distancia al objetivo.

Una de las características del mercado de trabajo europeo y español es el elevado desempleo de los jóvenes. El objetivo perseguido en términos de tasa de paro juvenil ya se logra a nivel nacional, y en el caso de Aragón y Baleares la situación es relativamente muy buena con casi un $13 \%$ de tasa de paro. En la posición opuesta están Extremadura, Canarias y Asturias que cuentan con tasas superiores al 23\%. De todas maneras los jóvenes son uno de los colectivos que sufren en mayor medida el desempleo, por ello habría que incidir en medidas para incrementar su empleo, como el fomento del contrato a tiempo parcial, la realización de prácticas, bonificaciones en su contratación, la mejora de su formación, etc.

En cuanto al objetivo de alcanzar el 50\% como tasa de empleo de las personas entre 55 y 64 años, y así conseguir una mayor permanencia en el mercado laboral, nuestro país se encuentra a casi tres puntos de este objetivo. Las Comunidades de Baleares, Cataluña, Madrid, Navarra y La Rioja superan ese umbral; mientras que Andalucía, Extremadura y Asturias son las regiones que más difícil tienen conseguir esa meta.

Por lo que respecta a la siniestralidad, el objetivo hace referencia a la reducción de la siniestralidad laboral en un $15 \%$ medida como índice de incidencia. En este sentido, las Comunidades que presentan una menor siniestralidad no coinciden con las que tienen mejores tasas de empleo. Los mejores resultados se presentan en Extremadura 
y Aragón, siendo regiones con situaciones laborales muy distintas, la primera con la menor tasa de empleo y Aragón con una de las mayores tasas de empleo. En el lado contrario, Castilla-La Mancha, Baleares, Canarias, Comunidad Valenciana y Andalucía presentan tasas de siniestralidad superiores a la media nacional.

En conclusión, el mercado de trabajo es el ámbito donde más avances se han registrado hacia los objetivos de Lisboa ${ }^{11}$, favorecido por la evolución del ciclo económico en España, que se encuentra en un largo período de expansión. Cabe señalar que las Comunidades que están mejor situadas y las Comunidades peor situadas mantienen sus posiciones en la batería de indicadores, salvo en el caso de la siniestralidad, donde se mezclan regiones con tasas de empleo muy diferentes. Cuatro comunidades (Baleares, Cataluña, Madrid y Navarra) cumplen los tres objetivos originales de la Estrategia de Lisboa $^{12}$.

\section{Indicadores de la educación, I+D, nuevas tecnologías}

El análisis se va a centrar, en primer lugar, en los indicadores relativos al capital humano. Por lo que respecta al objetivo de escolarización a los dos años (del 30\%), éste se alcanza en el País Vasco, Cataluña, Aragón y Madrid, mientras que los peores datos se localizan en Canarias, Navarra, Extremadura, Asturias y La Rioja con tasas inferiores al $5 \%$.

La media nacional en cuanto a la tasa bruta de graduación en ESO está a casi diez puntos del objetivo pretendido. Por encima de la media se encuentra Asturias, País Vasco, Navarra, Cantabria, Castilla y León, Galicia, Aragón, Cataluña y Madrid, siendo las dos primeras regiones las únicas que superan el objetivo. Los dos archipiélagos son los que en peor situación se encuentran (en torno al 60-65\% de graduados).

Para evitar que los alumnos abandonen de forma prematura sus estudios, dado que se considera esencial para una efectiva participación en la formación permanente

${ }^{11}$ La Comisión Europea reconoce que en España se ha avanzado satisfactoriamente en cuanto al empleo femenino; sin embargo, se observan en el mercado laboral español ciertas deficiencias que deben corregirse como es su persistente segmentación. Además considera que se ha de modernizar la protección del empleo e incrementar el atractivo del trabajo a tiempo parcial.

${ }^{12}$ Una tasa de empleo global del 70\%, una tasa de empleo femenino del $60 \%$ y una tasa de empleo de los trabajadores mayores (de 55 a 64 años) del 50\% para 2010. 
como mecanismo que garantiza la inserción en el mercado de trabajo, se pretende que dicha tasa de abandono sea del $15 \%$. Objetivo para el que hay que tomar determinadas medidas pues la situación de nuestro país no es buena, duplica en 2005 ese objetivo $^{13}$. Sólo en el País Vasco el estado de este indicador es mejor que el deseado, encontrándose relativamente cerca de ese porcentaje Navarra y Asturias. Sin embargo, en muchas regiones el abandono escolar prematuro es un problema, especialmente en Baleares, Murcia, Andalucía, Extremadura y Castilla-La Mancha, con tasas que superan el $35 \%$.

La importancia del nivel educativo para la proyección profesional del joven se constata en el objetivo de que al menos el $80 \%$ de los jóvenes de 20 a 24 años hayan completado el segundo ciclo de la educación secundaria. Se estima la necesidad de poseer este nivel educativo para poder intervenir íntegramente en los procesos de formación permanente y así lograr el desarrollo de una carrera profesional. España se encuentra muy lejos de lograrlo (casi a 20 puntos). Sólo el País Vasco lo cumple, encontrándose Navarra a 3 puntos de ese porcentaje. El resto de las regiones se sitúan a una distancia entre 7 y casi 30 puntos, estando Baleares, Murcia, Andalucía y Extremadura a la cola.

Un indicador de la modernización de la enseñanza superior, indispensable para convertirnos en una economía del conocimiento, es un aumento del 15\% del número de graduados en ciencias, matemáticas y tecnología de aquí al 2010. España se encuentra sólo a un poco más de un punto de esa meta. Son muchas las Comunidades que ya han conseguido el objetivo perseguido en el PNR (13,5\%), sobresaliendo el País Vasco, con un 25,20\% de graduados. Junto a esta Comunidad, Castilla y León, Asturias, Aragón, Galicia y Cantabria superan el objetivo establecido en la Estrategia de Lisboa (15\%). Las regiones peor situadas son los dos archipiélagos, Baleares y Canarias.

Dado el papel crucial que se otorga a la formación permanente en la actualización y mejora de las cualificaciones de los individuos a lo largo de su vida laboral y así

${ }^{13}$ En la Comunicación de la Comisión al Consejo Europeo de Primavera COM (2006) 816 final La Ejecución en España de la Estrategia de Lisboa Renovada para el Crecimiento y el Empleo para el caso español, la Comisión Europea considera que se tiene que producir más mejoras en educación y formación, recomendando que se produzcan reformas educativas efectivas, también a nivel regional, con la finalidad de reducir el abandono prematuro de los estudios. De ahí que estime que se debe prestar especial atención a la aplicación de la nueva ley de educación (Ley Orgánica 2/2006, de 3 de mayo, de Educación) para el logro de dicho objetivo. 
hacer frente a las necesidades del mercado laboral en un entorno cada vez más variable, se establece que el $12,5 \%$ de las personas de 25 a 64 años haya cursado recientemente algún tipo de estudios hasta el 2010. La media nacional está muy próxima al objetivo, y las distintas Comunidades muestran valores similares, no habiendo tanta distancia entre ellas. Las regiones mejor y peor ubicadas son Navarra $(16,30 \%)$ y Murcia $(9,50 \%)$, respectivamente.

En el ámbito de la I+D y las nuevas tecnologías, Madrid, Navarra, País Vasco y Cataluña son las regiones que realizan un mayor esfuerzo en actividades de I+D (como porcentaje del PIB), además son las únicas que superan la media nacional, estando Madrid muy cerca del objetivo del PNR del 2\% del PIB ${ }^{14}$. Baleares, Castilla-La Mancha y Cantabria se encuentran en una posición bastante complicada, su gasto en I+D es inferior al 0,5\% de su PIB. La iniciativa privada participa en este ámbito superando el objetivo propuesto del $55 \%$ en el País Vasco, Cataluña, Navarra, Castilla-La Mancha, La Rioja y Aragón y en el caso de las dos primeras se alcanza el objetivo propuesto por la Estrategia de Lisboa de que las 2/3 partes del gasto en I +D se sufrague por el sector privado. La media nacional está muy cerca del objetivo del PNR, lo mismo que la Comunidad de Madrid; mientras que Galicia y Baleares son las regiones más alejadas.

En lo relativo a las nuevas tecnologías, que permiten la mejora de la eficiencia y de la productividad, el objetivo de que el 30\% de los hogares cuenten con Internet es ampliamente superado por todas las Comunidades, excepto por Galicia y Extremadura. En Cataluña y País Vasco casi la mitad de los hogares tiene conexión a Internet, mientras que Madrid supera esa proporción.

En cuanto al acceso de Internet en los centros educativos, el objetivo de que el $100 \%$ de los centros cuenten con Internet es algo que próximamente va a alcanzarse,

${ }^{14}$ Como señala la Comunicación COM (2006) 816 final, se ha producido en los Estados miembros una reorientación de políticas hacia la investigación y la innovación; se reconoce la aplicación o la puesta a punto de reformas prometedoras. Un mayor esfuerzo en I+D contribuiría a un aumento del potencial de crecimiento económico de la Unión Europea. La solución radica en la mayor participación privada en ese gasto, para lo que es necesario «la vinculación entre ciencia e industria, promover conglomerados industriales competitivos a nivel internacional, mejorar el acceso a la financiación y aumentar los servicios de apoyo a la innovación». Para el caso español, se fija que entre los puntos fuertes en la ejecución de su PNR se encuentran buenos avances en la aplicación del plan de investigación, desarrollo e innovación. 
la media nacional se encuentra en el 98,5\%, y ya se logra en Aragón y Navarra. La situación de Galicia llama la atención, sólo el 88,4\% centros cuentan con conexión a Internet.

Cabe concluir que determinadas Comunidades alcanzan casi todos los objetivos relativos al capital humano y tecnológico, destacando el País Vasco. Por el contrario, otra Comunidad del norte del país, Galicia, se encuentra en los últimos lugares de todos los indicadores.

En la evaluación del PNR del Gobierno español, respecto a estos indicadores se considera que España ha avanzado en la aplicación del plan de investigación, desarrollo e innovación y en la mejora de las tasas de empleo, en especial del empleo femenino. Sin embargo, en ese análisis se pone de manifiesto que se debe reducir el problema de la segmentación del mercado laboral español y avanzar en más mejoras en materia de educación y formación.

\section{Principales Resultados}

Uno de los objetivos que se había propuesto en la elaboración de este trabajo es el diseño de un instrumento que permita evaluar el progreso en el cumplimiento de los objetivos de la Estrategia de Lisboa en las distintas Comunidades Autónomas en materia de empleo. Con este objetivo en mente, tal como se ha comentado en el apartado de selección de variables, se ha procedido a seleccionar una batería de indicadores relativos al mercado laboral, al capital humano y a la inversión en I+D.

En el desarrollo de este apartado se va a diseñar, en primer lugar, un indicador de cumplimiento global de los objetivos de Lisboa en materia laboral y de capital humano (IG) y luego se procederá a estimar indicadores individualizados para el capital humano $^{15}$ (ICH) y para el área del mercado de trabajo (IL).

Cabe recordar que el cálculo de la $\mathrm{DP}_{2}$ exige una jerarquización del orden de entrada de las variables y cálculos interactivos, hasta que exista una convergencia entre dos resultados sucesivos.

A) Indicador de cumplimiento global de los objetivos de Lisboa en materia laboral y de capital humano $(I G)$ :

${ }^{15}$ Dentro de este área se ha incluido la inversión en I+D. 
En la segunda columna del cuadro siguiente se recoge el orden de entrada de las variables en nuestro indicador sintético global (IG), que viene determinado por el valor de los coeficientes de correlación en valor absoluto de las variables con el valor de la $\mathrm{DP}_{2}$ retenido en la iteración anterior a alcanzar la convergencia. En la tercera columna aparece la información sobre el factor corrector, que nos indica el porcentaje de información nueva que incorpora la nueva variable al entrar en el indicador sintético, descontando el efecto de las variables ya agregadas previamente al indicador.

Cuadro 2. Estructura del indicador sintético de cumplimiento global (IG)

\begin{tabular}{|l|c|c|}
\hline INDICADOR GLOBAL (IG) & $|r|$ & $\left(1-R^{2}\right)$ \\
\hline PARTICIPACIÓN PRIVADA I+D & 0.7297 & 1 \\
\hline NIVEL DE ESTUDIOS & 0.7254 & 0.7289 \\
\hline GRADUADOS EN CIENCIAS, MATEMÁTICAS Y TECNOLOGÍA & 0.7086 & 0.3418 \\
\hline GASTO I+D/PIB & 0.7004 & 0.5372 \\
\hline TASA BRUTA DE GRADUACIÓN EN ESO & 0.6511 & 0.1949 \\
\hline ABANDONO ESCOLAR PREMATURO & 0.6418 & 0.0203 \\
\hline TASA DE EMPLEO FEMENINO & 0.6287 & 0.0617 \\
\hline TASA DE EMPLEO & 0.5847 & 0.2833 \\
\hline SINIESTRALIDAD & 0.5096 & 0.1512 \\
\hline TASA DE DESEMPLEO JUVENIL & 0.5029 & 0.3014 \\
\hline ESCOLARIZACIÓN A LOS 2 AÑOS & 0.4900 & 0.4711 \\
\hline TASA OCUPACIÓN MAYORES & 0.4119 & 0.3919 \\
\hline FORMACIÓN PERMANENTE & 0.4115 & 0.4961 \\
\hline INTERNET VIVIENDAS & 0.3843 & 0.2422 \\
\hline INTERNET CENTROS EDUCATIVOS & 0.3501 & 0.3323 \\
\hline
\end{tabular}

Fuente: Elaboración propia.

Los primeros indicadores que forman parte de nuestro indicador guardan relación con la inversión en I+D y con la educación.

El primer indicador en acceder al indicador sintético es el porcentaje de gasto en I+D realizado por las empresas y que retiene el $100 \%$ de información, le sigue el nivel de estudios con un factor corrector muy reducido del 4\%. A continuación, la tasa de graduados en ciencias, matemáticas y tecnología, que retiene un porcentaje de información del 34\% y el porcentaje de gastos en I+D sobre el PIB que retiene un $53 \%$ de información, en ambos casos descontando el efecto de las variables ya incorporadas al 
indicador sintético. Estos cuatro indicadores presentan altas correlaciones con el indicador sintético superiores en todos los casos al $70 \%$.

Después acceden al indicador un par de indicadores de tipo educativo como son la tasa bruta de graduación en ESO y la tasa de abandono escolar prematuro, que presentan factores correctores reducidos del $19 \%$ y del $2 \%$ respectivamente.

Los siguientes indicadores parciales en incorporarse son indicadores relacionados con el mercado laboral como son: la tasa de empleo femenino, la tasa de empleo total, la siniestralidad y la tasa de desempleo juvenil. Estos indicadores presentan correlaciones moderadas comprendidas entre un 50 y $63 \%$ y unos factores correctores bastante reducidos.

Los últimos cinco indicadores en acceder al indicador sintético son la tasa de escolarización a los dos años, la tasa de ocupación de los mayores, la formación permanente y dos indicadores de tipo tecnológico como son el acceso a Internet de las

Cuadro 3. Resultados del indicador sintético global (IG)

\begin{tabular}{|l|c|c|}
\hline & INDICADOR GLOBAL (IG) & ORDENACIÓN \\
\hline MADRID (Com. de) & 4.7028 & 1 \\
\hline NAVARRA (C. Foral de) & 5.0275 & 2 \\
\hline PAIS VASCO & 5.0317 & 3 \\
\hline ARAGON & 5.4064 & 5 \\
\hline CATALUÑA & 7.029 & 6 \\
\hline CASTILLA Y LEON & 7.7613 & 7 \\
\hline RIOJA (La) & 8.4641 & 8 \\
\hline COMUNIDAD VALENCIANA & 9.4204 & 10 \\
\hline ASTURIAS (Ppdo. de) & 10.5147 & 11 \\
\hline CANTABRIA & 10.6281 & 12 \\
\hline GALICIA & 11.5084 & 13 \\
\hline MURCIA (Región de) & 11.708 & 14 \\
\hline CASTILLA-LA MANCHA & 12.3112 & 15 \\
\hline CANARIAS & 13.3205 & 16 \\
\hline ANDALUCIA & 13.7505 & 17 \\
\hline BALEARS (Illes) & 14.3011 & \\
\hline EXTREMADURA & 15.9898 & 8 \\
\hline ESPAÑA & 8.6612 & \\
\hline
\end{tabular}

Fuente: Elaboración propia. 
viviendas y el acceso a Internet de los centros educativos. En todos los casos el volumen de información que retienen está comprendido entre un 25 y un 50\% aproximadamente descontando el efecto de las variables previamente incorporadas al indicador sintético.

A continuación, se presenta en el cuadro 3 el valor del indicador sintético para las diferentes Comunidades Autónomas, apareciendo también el dato de España como agregado. Se recoge en la primera columna el valor de la $\mathrm{DP}_{2}$ por Comunidades Autónomas y en la segunda la posición que ocupa en el ranking total. Aquellas Comunidades Autónomas con un valor del indicador de cumplimiento más reducido están más próximas a los objetivos que aquellas que tienen un valor más elevado. En este caso encabezan el ranking Madrid, Navarra, País Vasco, Aragón y Cataluña con valores del indicador inferiores al dato total para el agregado nacional. Al otro lado de la tabla se encuentran Extremadura, Baleares, Andalucía, Canarias y Castilla-La Mancha con valores del indicador superiores a 12 .

En términos espaciales se observa como la zona Norte-Levante junto a Madrid son los que están más cerca de cumplir los objetivos, siendo esta última Comunidad la que obtiene mejores valores del indicador sintético. También se puede apreciar cierto grado de polarización del Norte frente al Sur y al territorio Insular.

A continuación, se comentan los resultados para el indicador capital humano (ICH) y posteriormente para el indicador laboral (IL).

Cuadro 4. Estructura del indicador sintético capital humano (ICH)

\begin{tabular}{|l|c|c|}
\hline INDICADOR DE CAPITAL HUMANO (ICH) & $|\mathrm{r}|$ & $\left(1-\mathrm{R}^{2}\right)$ \\
\hline NIVEL DE ESTUDIOS & 0.8168 & 1 \\
\hline $\begin{array}{l}\text { GRADUADOS EN CIENCIAS, MATEMÁTICAS } \\
\text { Y TECNOLOGÍA }\end{array}$ & 0.7962 & 0.0429 \\
\hline TASA BRUTA DE GRADUACIÓN EN ESO & 0.7945 & 0.3540 \\
\hline ABANDONO ESCOLAR PREMATURO & 0.7906 & 0.0666 \\
\hline GASTO I+D/PIB & 0.7489 & 0.5599 \\
\hline PARTICIPACIÓN PRIVADA I+D & 0.7052 & 0.7012 \\
\hline FORMACIÓN PERMANENTE & 0.4278 & 0.3312 \\
\hline ESCOLARIZACIÓN A LOS 2 AÑOS & 0.4109 & 0.6628 \\
\hline INTERNET CENTROS EDUCATIVOS & 0.3188 & 0.8015 \\
\hline INTERNET VIVIENDAS & 0.2580 & 0.4357 \\
\hline
\end{tabular}

Fuente: Elaboración propia. 


\section{B) Indicador de capital humano (ICH):}

Los primeros indicadores en formar parte del indicador ICH son el nivel de estudios junto a la tasa de graduados en ciencias, matemáticas y tecnología. Le siguen los indicadores tasa de graduación en ESO y tasa de abandono escolar prematuro junto a los indicadores relacionados con la inversión en I+D y con la formación permanente. De nuevo, los indicadores relacionados con el acceso a Internet ocupan las últimas posiciones en el ranking, al igual que la tasa de escolarización a los dos años.

En cuanto a los factores correctores de los diferentes indicadores parciales, estos son superiores al 35\% para todos los indicadores, a excepción del porcentaje de graduados en ciencias, matemáticas y tecnología y de la tasa de abandono escolar prematuro cuyos valores son inferiores al $6 \%$.

Cuadro 5. Resultados del indicador sintético de capital humano (ICH)

\begin{tabular}{|c|c|c|c|}
\hline & $\begin{array}{c}\text { INDICADOR } \\
\text { CAPITAL } \\
\text { HUMANO (ICH) }\end{array}$ & ORDENACIÓN & $\begin{array}{c}\text { DIFERENCIA } \\
\text { ORDENACIÓN } \\
\text { IG-ICH }\end{array}$ \\
\hline PAIS VASCO & 0.9677 & 1 & 2 \\
\hline MADRID (Com. de) & 2.7489 & 2 & -1 \\
\hline NAVARRA (C. Foral de) & 2.9463 & 3 & -1 \\
\hline ARAGON & 3.2733 & 4 & 0 \\
\hline CATALUÑA & 4.7763 & 5 & 0 \\
\hline CASTILLA Y LEON & 4.9427 & 6 & 0 \\
\hline ASTURIAS (Ppdo. de) & 5.4555 & 7 & 2 \\
\hline RIOJA (La) & 6.4355 & 8 & -1 \\
\hline COMUNIDAD VALENCIANA & 6.9408 & 9 & -1 \\
\hline CANTABRIA & 7.1226 & 10 & 0 \\
\hline MURCIA (Región de) & 8.4185 & 11 & 1 \\
\hline ANDALUCIA & 8.9422 & 12 & 3 \\
\hline CASTILLA-LA MANCHA & 8.9967 & 13 & 0 \\
\hline CANARIAS & 9.4964 & 14 & 0 \\
\hline GALICIA & 9.8073 & 15 & -4 \\
\hline BALEARS (Illes) & 11.193 & 16 & 0 \\
\hline EXTREMADURA & 11.6992 & 17 & 0 \\
\hline ESPAÑA & 6.7336 & & \\
\hline
\end{tabular}

Fuente: Elaboración propia. 
Los resultados de este indicador por Comunidades Autónomas nos sitúan en una situación similar a la del indicador global, donde se aprecian pequeñas diferencias de variación en el ranking con respecto al obtenido para IG. Las variaciones más importantes a nivel regional son las de Andalucía que mejora en tres posiciones y Galicia que empeora en cuatro. Esta información puede consultarse en el cuadro 5. En la primera columna se recoge el resultado del indicador, en la segunda la ordenación en el ranking de las diferentes Comunidades Autónomas respecto al resultado de este indicador y en la última la diferencia de las ordenaciones de los indicadores IG e ICH.

De nuevo se observa un escenario con cierto grado de polarización del Norte frente al Sur y al territorio Insular.

\section{C) Indicador laboral (IL):}

En cuanto a nuestro indicador laboral (IL), tal y como se puede ver en el cuadro siguiente, los primeros indicadores parciales en formar parte del indicador sintético son las diferentes tasas de empleo, con valores de los coeficientes de correlación superiores al $69 \%$, y con volúmenes de información del $100 \%$ para la tasa de empleo femenino, del $21 \%$ para las tasas de empleo total y la tasa de ocupación de los mayores y del $31 \%$ para la tasa de desempleo juvenil, en todos los casos descontando el efecto de las variables previamente ya incorporadas al indicador. La tasa de siniestralidad entra en el último lugar en nuestro indicador sintético con un factor corrector muy elevado de un $80 \%$ y un coeficiente de correlación nada despreciable del 0.58 .

Cuadro 6. Estructura del indicador laboral (IL)

\begin{tabular}{|l|c|c|}
\hline INDICADOR LABORAL (IL) & $|r|$ & $\left(1-R^{2}\right)$ \\
\hline TASA DE EMPLEO FEMENINA & 0.8469 & 1 \\
\hline TASA DE EMPLEO & 0.746 & 0.2176 \\
\hline TASA OCUPACIÓN MAYORES & 0.6941 & 0.2195 \\
\hline TASA DE DESEMPLEO JUVENIL & 0.6900 & 0.3137 \\
\hline SINIESTRALIDAD & 0.5883 & 0.8083 \\
\hline
\end{tabular}

Fuente: Elaboración propia.

El resultado por Comunidades nos sitúa en un escenario más disperso en donde no se observa el patrón de enfrentamiento zona Norte-Levante frente a Sur-Islas. Además se producen importantes variaciones en el ranking con respecto al IG, tal y 
como se observa en el cuadro 7. Asturias y País Vasco descienden 5 y 6 posiciones en el ranking frente a Galicia y Baleares que mejoran en 5 y 4 posiciones, respectivamente.

En este caso las regiones que obtienen mejores resultados son Aragón, Madrid, Navarra, La Rioja y Cataluña, frente a Andalucía, Castilla-La Mancha, Extremadura, Asturias y Canarias que se sitúan en las últimas posiciones.

Cuadro 7. Resultados del indicador laboral (IL)

\begin{tabular}{|c|c|c|c|}
\hline & $\begin{array}{c}\text { INDICADOR } \\
\text { CAPITAL } \\
\text { HUMANO (ICH) }\end{array}$ & ORDENACIÓN & $\begin{array}{c}\text { DIFERENCIA } \\
\text { ORDENACIÓN } \\
\text { IG-IL }\end{array}$ \\
\hline ARAGON & 2.9118 & 1 & 3 \\
\hline MADRID (Com. de) & 3.416 & 2 & -1 \\
\hline NAVARRA (C. Foral de) & 3.603 & 3 & -1 \\
\hline RIOJA (La) & 3.7532 & 4 & 3 \\
\hline CATALUÑA & 3.7673 & 5 & 0 \\
\hline GALICIA & 4.6895 & 6 & 5 \\
\hline CASTILLA Y LEON & 4.7002 & 7 & -1 \\
\hline CANTABRIA & 4.7115 & 8 & 2 \\
\hline PAIS VASCO & 5.0508 & 9 & -6 \\
\hline COMUNIDAD VALENCIANA & 5.2136 & 10 & -2 \\
\hline MURCIA (Región de) & 5.5573 & 11 & 1 \\
\hline BALEARS (Illes) & 5.6697 & 12 & 4 \\
\hline CANARIAS & 7.4173 & 13 & 1 \\
\hline ASTURIAS (Ppdo. de) & 7.6491 & 14 & -5 \\
\hline EXTREMADURA & 8.3339 & 15 & 2 \\
\hline CASTILLA-LA MANCHA & 8.8709 & 16 & -3 \\
\hline ANDALUCIA & 8.9784 & 17 & -2 \\
\hline ESPAÑA & 5.0821 & & \\
\hline
\end{tabular}

Fuente: Elaboración propia. 
6. Discusión del CONJUNTO de RESUltados E IMPLICACIONES PARA LA POLÍtiCA ECONÓMiCA

Más allá de los análisis puramente cuantitativos de los resultados de nuestros indicadores sintéticos y de la situación de las diferentes Comunidades Autónomas en relación a los indicadores parciales, nos permitimos a continuación formular algunas reflexiones y recomendaciones en materia de política de empleo con el objetivo de recortar las distancias de aquellas Comunidades más desfavorecidas en relación con los objetivos de la Estrategia de Lisboa, aunque anteriormente ya se han sugerido ciertas líneas de actuación.

Nuestro análisis ha puesto de manifiesto cómo existen grandes disparidades regionales en materia laboral, educación e I+D, disparidades que vienen explicadas en cierta manera por las peculiaridades de cada Comunidad de tipo territorial, sectorial, poblacional... y que hacen casi imposible formular un único tipo de política con el fin de acortar esas distancias.

En la Estrategia Renovada de Lisboa se hace un claro llamamiento a que los diferentes Estados miembros intensifiquen sus esfuerzos en materia de empleo, en particular, aplicando políticas activas de empleo, centrándose entre otras prioridades en dar oportunidades a los jóvenes, hacer frente al desempleo estructural y a la baja calidad del empleo, garantizar la formación y la igualdad de oportunidades. Sin duda alguna nos enfrentamos al gran reto de adecuar nuestras condiciones laborales, tanto de nuestro país como de las Comunidades Autónomas, en un ámbito donde la sociedad del conocimiento nos enfrenta a grandes retos.

En un contexto de descentralización en la gestión de estas políticas, las regiones asumen nuevas competencias; sin embargo, los resultados de su aplicación han sido muy desiguales, sin duda por la acción de diferentes factores y por la propia dinámica de sus mercados de trabajo. Además nos permitimos sugerir que para lograr mayores niveles de eficacia y eficiencia en las políticas de empleo se requiere una mayor coordinación entre las distintas administraciones públicas que ejecutan estas políticas.

A la vista de los resultados de nuestro indicador global las diferentes Comunidades deberían potenciar la inversión en $\mathrm{I}+\mathrm{D}$, junto a la enseñanza reglada como mecanismos que garanticen la inserción laboral en una sociedad de la información, así como el incremento del empleo estable dando preferencia a los colectivos que tradicionalmente tienen mayores dificultades de acceso al mercado de trabajo como los mayores de 45 años, los jóvenes o las mujeres.

El crecimiento de la economía española se ha basado fundamentalmente en el uso más intensivo de la mano de obra, manifestándose en tasas de crecimiento del empleo 
importantes. A pesar de ello, las tasas de empleo femenino siguen por debajo de la media comunitaria, por lo que es necesario determinadas reformas para mejorar esta situación. El logro de una adecuada conciliación de la vida familiar y profesional exigen el establecimiento de mecanismos necesarios que permitan, por un lado, disminuir las situaciones de salida del mercado laboral como consecuencia de la necesidad de atender las responsabilidades familiares (en especial, el cuidado de menores de seis años y de personas dependientes) y, por otro, respaldar la decisión de la persona a la hora de elegir la forma de compatibilizar la vida laboral y personal. Las iniciativas deben ir concretamente orientadas a promover la dotación de servicios de apoyo familiar y a favorecer, por parte de las empresas, la flexibilidad de la jornada y del horario laborales y la concesión de permisos y licencias para el cuidado de los menores. A pesar de los avances producidos en la escolarización en los últimos años, la existencia de servicios asequibles de atención y cuidado de los menores se convierte en una de las mayores demandas para resolver el problema de la conciliación. El indicador de escolarización a los dos años es muy bajo en Extremadura y Asturias, regiones con bajas tasas de empleo femenino (caso contrario al de Navarra y la Rioja, con elevadas tasas). También hay que esperar a los efectos del desarrollo de la Ley Orgánica 3/2007, de 22 de marzo, para la igualdad efectiva de mujeres y hombres.

Ante la evolución demográfica y el proceso de envejecimiento de la sociedad europea, se pretende prolongar la permanencia laboral de las personas, y así garantizar la sostenibilidad futura de los sistemas de protección social, lo que requiere medidas que proporcionen un entorno laboral atractivo y adaptable, unas buenas condiciones de seguridad en el trabajo, la mejora del acceso a la formación, formas flexibles de organización del trabajo, políticas de mercado de trabajo activas, adecuados incentivos financieros y mejora de la calidad del empleo.

Aunque se va en buena dirección en la mejora de los índices de empleo, una cuestión que es de máxima prioridad en el debate europeo es la calidad de los empleos creados. La mejora de la calidad y la productividad laboral constituyen uno de los objetivos generales en las Directrices de empleo junto con el pleno empleo y la cohesión social. Por tanto, la mejora de la calidad del empleo constituye uno de los grandes retos en los próximos años.

Llama la atención el elevado abandono prematuro de los estudios y el bajo porcentaje de jóvenes de 20 a 24 años que han completado el segundo ciclo de la educación secundaria en Baleares, lo que en principio no afecta a su inserción al mercado laboral, pues presenta la tasa de paro juvenil más baja. Situación contraria a la de Asturias, con una elevada tasa de paro y un bajo porcentaje de abandono escolar, lo que puede estar relacionado con su escaso tejido productivo, esto es, los jóvenes deci- 
den terminar la formación necesaria para poder encontrar un empleo, pero aún así lo tienen difícil.

También se observa que la zona norte presenta la mayor proporción de graduados en ciencias, matemáticas y tecnología, es decir se forman pero luego emigran hacia otras Comunidades Autónomas por falta de empresas donde desarrollar su carrera profesional.

Para continuar en el proceso de convergencia real, a medio y largo plazo se debe mejorar la productividad, cuestión que se enfatiza en el PNR español. La inversión en I+D+i es el terreno donde los indicadores están más alejados de los objetivos, de ahí que se debe fomentar esta inversión, sobre todo, en el sector privado; detrás de esto puede estar la dimensión de las empresas españolas, con un claro predominio de las PYMES.

\section{Conclusiones}

El presente trabajo ha perseguido el diseño de indicadores sintéticos de cumplimiento regional de los objetivos de la Estrategia de Lisboa en materia de empleo, educación e inversión en I+D, con el fin de analizar la situación de las diferentes Comunidades Autónomas, para lo que se ha utilizado un conjunto de 16 indicadores.

En cuanto a los resultados, en todos los indicadores sintéticos las Comunidades Autónomas que se sitúan a la cabeza son Aragón, Navarra, Madrid, Cataluña y País Vasco (excepto esta última Comunidad en el indicador IL). Los resultados del indicador global (IG) y del indicador de capital humano (ICH) son similares, apreciándose cierto enfrentamiento de las regiones del Norte de España frente a las de Sur. Respecto al indicador sintético laboral (IL), se observa un patrón geográfico más difuso, no se aprecia la existencia de polarización entre la zona Norte-Levante frente al Sur-Islas.

El mercado de trabajo es el ámbito donde más avances se han registrado hacia los objetivos de Lisboa, favorecido por la evolución del ciclo económico. Las Comunidades mejor y peor situadas mantienen sus posiciones en el conjunto de indicadores, salvo en el caso de la siniestralidad. Baleares, Cataluña, Madrid y Navarra cumplen ya los tres objetivos originales de la Estrategia de Lisboa.

Es interesante destacar la buena situación del País Vasco en los objetivos relativos al capital humano y tecnológico; mientras que Galicia ocupa los últimos lugares en todos los indicadores. En los indicadores de capital humano, tanto los dos archipiélagos (sobre todo, las Islas Baleares) como Murcia son las regiones que presentan 
unos resultados desfavorables. En I+D la región peor situada es Galicia, acompañada por las Islas Baleares (en porcentaje del gasto en I+D y sobre la participación de las empresas). Llama la atención, por tanto, la posición desfavorable de las Islas Baleares en los indicadores de I+D y de capital humano, a pesar de ser una de las Comunidades con mayor PIB per cápita ${ }^{16}$, lo que puede estar relacionado con su especialización productiva orientada hacia el sector servicios y, más concretamente, al sector turístico.

Más allá de la situación actual de las diferentes Comunidades Autónomas respecto a los objetivos de Lisboa, es necesario plantearnos de cara al futuro un análisis temporal que nos permita evaluar los esfuerzos que han efectuado las distintas Comunidades Autónomas en su camino a lograr los objetivos, pues su situación de partida es muy diferente.

\section{BiblografíA}

Comisión de las Comunidades Europeas (2005), «Working together for the growth and jobs. A new start for the Lisbon Strategy», Communication to the Spring European Council, Brussels.

- (2006), 816 final Ejecución de la Estrategia de Lisboa Renovada para el Crecimiento y el Empleo, Comunicación de la Comisión al Consejo Europeo de Primavera, Bruselas.

- (2007), 61 final Un marco coherente de indicadores y puntos de referencia para el seguimiento de los avances hacia los objetivos de Lisboa en el ámbito de la educación y la formación, Bruselas.

De la Fuente, A. y Estrada, A. (2005), «Indicadores de cumplimiento regional de los objetivos de Lisboa. Metodología, Fuentes y Resultados», Documento de Trabajo Dirección General de Análisis y Programación Presupuestaria, D-2006-01.

GonzÁlez, J. M. y López, P. (2005), «El relanzamiento de la estrategia de Lisboa», Boletín Económico del Banco de España, núm. 6/2005, 75-91, Madrid.

Merino, M. C. y Somarriba, N. (2007), «Evaluación de los objetivos de empleo de la Estrategia de Lisboa en las Comunidades Autónomas. Un análisis comparativo», Actas de la XXXIII Reunión de Estudios Regionales, León.

Montalvo, A. (2005), «La Estrategia de Lisboa: de la política económica a la economía política», Información Comercial Española (ICE), núm. 826, 503-527.

${ }^{16}$ El PIB per cápita de las Islas Baleares en 2006 es de 24.456 euros, por encima de la media nacional y muy cerca de la media de la UE-25. 
Mulas, C. (2007), «La Estrategia de Lisboa, el Programa Nacional de Reformas y las políticas de oferta en España», Información Comercial Española (ICE), núm. 837, 231-245.

Oficina Económica del Presidente (2006), Programa Nacional de Reformas. Informe Anual de Progreso 2006.

Osuna, J. L. y Bueno C. (2007), «La evaluación de las políticas públicas: el caso de las políticas de fomento al empleo estable», Información Comercial Española (ICE), núm. 836, 75-83.

Pena, J. B. (1977), Problemas de la medición del bienestar y conceptos afines. Una aplicación al Caso Español, INE, Madrid.

Somarriba, N. y Pena, J. B. (2007), «Aproximación a un indicador regional y nacional de los objetivos de Lisboa a partir de la Medida de Distancia $\mathrm{P}_{2} »$, Anales de Economía Aplicada 2007, núm. XXI, Madrid, ASEPELT y Delta Publicaciones, 467.

ZARzosa, P. (1996), Aproximación a la medición del bienestar social, Valladolid, Secretariado de Publicaciones. 